\title{
Upregulated TRIM11 Exerts its Oncogenic Effects in Hepatocellular Carcinoma Through Inhibition of P53
}

\author{
Jinjin Liu ${ }^{a} \quad J u n$ Rao ${ }^{b} \quad$ Xuming Lou ${ }^{a} \quad J i a n$ Zhaic Zhenhua Nid Xiongbiao Wanga \\ aDepartment of Respiratory Medicine, Putuo Hospital, Shanghai University of Traditional Chinese \\ Medicine, Shanghai, bJiangxi Cancer Hospital, Nanchang, 'Department of Interventional Radiology, \\ Eastern Hepatobiliary Surgery Hospital, the Second Military Medical University, Shanghai, dCentral lab, \\ Putuo Hospital, Shanghai University of Traditional Chinese Medicine, Shanghai, China
}

\section{Key Words}

Trim11 • HCC $・$ P53 • Tumor growth $•$ Progression

\begin{abstract}
Background/Aims: The tripartite motif containing (TRIM) family plays crucial roles in tumor development and progression. However, little is known about the function and mechanism of TRIM11 in hepatocellular carcinoma (HCC). Methods: The expression levels of TRIM11 were examined by real-time PCR, Western blot and Immunohistochemical (IHC) staining. TRIM11 knockdown cells were produced by lentivirus infection, and functional assays, such as MTT, colony formation assay, migration and invasion assays and a xenograft tumor model were used to investigate the role of TRIM11 in HCC. We also determined the effect of TRIM11 on p53 signaling and its downstream molecules. Results: We found that TRIM11 mRNA and protein levels were significantly increased in HCC tissues as compared with normal tissues; increased levels correlated with poor patient survival. By loss- and gain-of-function investigations, knockdown of TRIM11 suppressed cell proliferation, migration, invasion in vitro and tumor growth in vivo. Moreover, TRIM11 negatively regulated p53 expression. Knockdown of p53 abrogated the in vitro and in vivo biological functions of TRIM11 shRNA in HCC cells. Conclusions: These data show that TRIM11 exerts its oncogenic effect in HCC by downregulating p53 both in vitro and in vivo. Our data provide new insights into the pathogenesis of HCC and indicate that TRIM11 may serve as a new therapeutic target for HCC treatment.

\section{Introduction}

Hepatocellular carcinoma (HCC) is the most common aggressive hepatic disease; it ranks as the fifth most common cancer and represents the third-leading cause of death from cancer

J. Liu and J. Rao contributed equally to this manuscript.

\begin{tabular}{ll}
\hline Jian Zhai, Zhenhua Ni and & Department of Interventional Radiology, Eastern Hepatobiliary Surgery Hospital, 2nd Military \\
Xiongbiao Wang & Medical University, Shanghai, (China); and Central lab \& Department of Respiratory Medicine, \\
& Putuo Hospital, Shanghai University of Traditional Chinese Medicine, Shanghai, (China) \\
& E-Mail jianzhai1979@126.com/zhenhuani@yeah.net/xiongbiao6@hotmail.com
\end{tabular}


worldwide [1]. The occurrence of HCC is highest in East and South-East Asia, especially in China, which alone accounts for approximately $50 \%$ of the total number of new liver cancer cases and deaths [2]. Despite the clinical implementation of various approaches, such as surgery, chemotherapy and radiotherapy, the 5-year survival rate for HCC remains relatively low [1]. Due to late stage detection and advanced metastatic disease as a result of spread via the lymphatic or blood system, HCC has a high mortality rate of over $90 \%[2,3]$. Consequently, identification of the role of dysregulated HCC-associated genes is urgently required to clarify the potential mechanism that regulates HCC development and progression, which may offer targets for the diagnosis, treatment and prognosis prediction of HCC.

Tripartite motif containing (TRIM) proteins have evolutionarily-conserved domain structures of a common really interesting new gene (RING) finger domain at the N-terminal, 1 or 2 B-box motifs and a coiled-coil (RBCC) region [4]. Most TRIM family proteins function as E3 ubiquitin ligases because of the RING finger domain [5]. Emerging evidence has demonstrated that TRIM family proteins play vital roles in innate immunity, cell proliferation, migration, differentiation and apoptosis, tumor development and progression [6-8]. TRIM11 is located on human chromosome 11 and has a PRY domain and a SPRY domain in addition to a RBCC domain. The importance of TRIM11 was first reported in nervous system function, such as in Alzheimer's disease, dopamine beta-hydroxylase expression, Pax6-dependent neurogenesis and neuron cell survival [9-12]. TRIM11 was also found to be important in IFN $\beta$ production and antiviral activity, restricting HIV-1 replication and autophagy [13-15]. Di and colleagues found overexpression of TRIM11 in high-grade gliomas and demonstrated that TRIM11 has an oncogenic function mediated through the epidermal growth factor receptor (EGFR) signaling pathway [16]. Recently, Wang et al. demonstrated that TRIM11 acts as an oncogene in lung cancer by promoting cell growth, migration and invasion [17]. Chen and colleagues found that overexpression of TRIM11 is closely associated with HCC progression and poor survival of the patients [18]. However, no investigation has been performed to explore the biological role of TRIM11 in HCC.

In the present study, we comprehensively investigated the biological function of TRIM11 and its underlying molecular mechanism in HCC. We found that TRIM11 expression levels were significantly increased in HCC tissues and cell lines. The increased TRIM11 levels correlated with hepatic cancer progression and poor patient survival. Knockdown of TRIM11 in HCC cell lines decreased proliferation, colony formation, migration, invasion in vitro and tumor growth in vivo. Moreover, TRIM11 negatively regulated p53 expression and knockdown of p53 abrogated the in vitro and in vivo biological functions of TRIM11 shRNA in HCC cells. Our data show an oncogenic role of TRIM11 in HCC development and progression, providing a potential therapeutic target for treatment of this disease.

\section{Materials and Methods}

\section{Clinical specimens and cell lines}

Ten fresh HCC tissue samples and matched adjacent normal liver tissues were collected from Eastern Hepatobiliary Surgery Hospital, the Second Military Medical University (Shanghai, China). All tissues were snap-frozen in liquid nitrogen immediately after surgery and stored at $-80^{\circ} \mathrm{C}$ until total RNA was extracted. A total of 60 paraffin-embedded HCC tissues and normal liver tissues collected between 2012 and 2016 were also obtained from our hospital. For the use of these clinical materials, written informed consent was obtained from all participating patients and the project was approved by the Ethics Committee of Eastern Hepatobiliary Surgery Hospital. The normal human hepatic cell line L02 and the HCC cell lines SMMC7721, MHCC97H, HepG2 and HCCLM3 were purchased from the Cell Resource Center, Chinese Academy of Science Committee (Shanghai, China). These cells were stored in liquid nitrogen and cultured in highglucose Dulbecco's modified Eagle's medium supplemented with 10\% FBS (GIBCO, Grand Island, NY, USA), 100 units $/ \mathrm{mL}$ penicillin and $100 \mathrm{~g} / \mathrm{mL}$ streptomycin in a humidified atmosphere of $5 \%(\mathrm{v} / \mathrm{v}) \mathrm{CO}_{2}$ in a $37^{\circ} \mathrm{C}$ incubator.

\section{KARGER}


RNA extraction and real-time PCR

Total mRNA was isolated from tissues and cells using TRIzol reagent (Invitrogen, Carlsbad, CA, USA). PrimeScript RT Reagent Kit (TaKaRa, Dalian, China) was used to synthesize complementary DNA (cDNA). Real-time PCR was performed using SYBR Premix Ex Taq II on an ABI 7900HT fast real-time PCR system (Applied Biosystems, Foster City, CA, USA). The sequences of primers used were as follows: TRIM11, forward 5'-GTAGAGACACTGCGGAGGTT-3',

reverse $5^{\prime}$-GACAGGATCAGCTCAGGGTT-3';

p53, forward 5'-CAGACCTATGGAAACTACTT-3',

reverse $5^{\prime}$-CTGCCCTGGTAGGTTTTCTG-3';

p21, forward $5^{\prime}$-TGGAGACTCTCAGGGTCGAAA-3',

reverse $5^{\prime}$-GGCGTTTGGAGTGGTAGAAATC- 3 ;

p27, forward 5'-AGTTAACCCGGGACTTGGAG-3',

reverse 5'-TCTGTAGTAGAACTCGGGCA-3';

CyclinD1, forward $5^{\prime}$-GCTGCGAAGTGGAAACCATC- 3 ',

reverse $5^{\prime}$-CCTCCTTCTGCACACATTTGAA- $3^{\prime}$. The relative mRNA expression levels of target genes were normalized to glyceraldehyde 3-phosphate dehydrogenase (GAPDH) and calculated by the $2^{-\Delta \triangle \mathrm{CT}}$ method. All PCR reactions were performed three times.

Plasmid construction and lentivirus production

The sequences of TRIM11-shRNA, p53-shRNA and scramble shRNA were cloned into the lentiviral vector pLK0.1. The cDNA of TRIM11 was cloned into the lentiviral expression vector pCDH-copGFP. Lentiviruses were produced by co-transfection of 293T cells with a lentiviral vector and packaging vectors according to the manufacturer's protocols. The lentiviral supernatant was collected to infect target cell lines in the presence of $8 \mu \mathrm{g} / \mathrm{mL}$ Polybrene (Sigma-Aldrich, St Louis, MO, USA).

\section{MTT and colony formation assays}

Cells were seeded into 96-well plates at a density of $3 \times 10^{3} / 100 \mu \mathrm{L} /$ well. After incubation for different times, $20 \mu \mathrm{L} 5 \mathrm{mg} / \mathrm{mL}$ MTT (Merck Millipore, Billerica, MA, USA) in PBS was added to each well and incubated at $37^{\circ} \mathrm{C}$ for another $4 \mathrm{~h}$. The supernatants were discarded and then $150 \mu \mathrm{L}$ DMSO was added to each well. The absorbance at $490 \mathrm{~nm}$ was measured using a microplate reader. The colony formation assay was performed using 500 cells/well seeded into six-well plates. After culture for 14 days, cells were fixed using $70 \%$ ethanol and stained with $0.1 \%$ crystal violet (Sigma-Aldrich) for counting.

\section{Migration and invasion assays}

The migration assay was performed using the Transwell system (24 wells, $8 \mu \mathrm{m}$ pore size with polycarbonate membrane) according to the manufacturer's protocols. Aliquots containing $5 \times 10^{4}$ cells/well were seeded into the upper chamber of plates with serum-free RPMI medium. RPMI medium supplemented with 10\% FBS was added to the lower compartment. After incubation for $48 \mathrm{~h}$, the cells remaining in the upper chamber were scraped out, and the cells on the lower side were fixed in methanol and stained with $0.1 \%$ crystal violet solution. To count the number of cells that had migrated to the lower side, five randomlyselected fields were photographed and counted under a light microscope. For invasion assays, $1 \times 10^{5}$ cells were seeded into the upper chambers coated with Matrigel@. The assay was then performed as for the migration assay.

\section{Immunohistochemical (IHC) staining}

For the immunohistochemical staining experiment, $5 \mu \mathrm{m}$ paraffin sections were baked for $2 \mathrm{~h}$ at $60^{\circ} \mathrm{C}$. Antigen retrieval was performed in sodium citrate buffer after deparaffinization and rehydration. Endogenous peroxidase activity was quenched in $3 \% \mathrm{H}_{2} \mathrm{O}_{2}$ in methanol, followed by blocking with $10 \%$ bovine serum albumin (BSA) in PBS for 60 min and incubating with primary antibodies at $4^{\circ} \mathrm{C}$ overnight. After three washes in PBS, sections were incubated with horseradish peroxidase (HRP)-conjugated secondary antibodies. The immune complex was visualized using DAB-chromogen (Dako, Glostrup, Denmark) according to the manufacturer's protocol. The nuclei were counterstained with hematoxylin. Quantitative analysis of the immunostained images was performed after color segmentation based on fixed 


\section{Cellular Physiology Cell Physiol Biochem 2017;44:255-266 \begin{tabular}{l|l|l} 
and Biochemistry & $\begin{array}{l}\text { DOI: 10.1159/000484678 } \\
\text { Published online: November 09, } 2017\end{array}$ & $\begin{array}{l}\text { C } 2017 \text { The Author(s). Published by S. Karger AG, Basel } \\
\text { www.karger.com/cpb }\end{array}$
\end{tabular}}

Liu et al.: Role of TRIM11 in HCC

threshold values of hue, saturation, and intensity. Each image was assigned a score calculated by multiplying the staining intensity by the area of positively-stained cells.

\section{Western blotting}

Tissues or cells were lysed using RIPA buffer (Sigma-Aldrich) with added protease inhibitor cocktail. Then, the amounts of protein in the lysates were measured using a BCA protein assay kit (Pierce, Rockford, IL, USA), followed by electrophoresis on an SDS-PAGE gel and transfer onto an Immobilon-P Transfer Membrane (Merck-Millipore, Darmstadt, Germany). The membranes were blocked with 10\% BSA, then incubated with primary antibody at $4^{\circ} \mathrm{C}$ overnight, followed by incubation with HRP-conjugated secondary antibody at room temperature for $1 \mathrm{~h}$. Signals were detected after chemiluminescence reaction with HRP Substrate (Merck-Millipore). Quantification of the intensity of blots was performed by Quantity One software (Bio-Red, Hercules, CA, USA). GAPDH was used as a control.

Tumor xenograft model

Cells were resuspended at a density of $1 \times 10^{7}$ cells $/ \mathrm{mL}$. A total of $100 \mu \mathrm{L}$ of cell suspensions mixed with Matrigel $\AA$ at a 1:1 ratio was injected subcutaneously into the right flanks of 4 week-old BALB/c nude mice (SLAC, Shanghai). The tumor size was monitored every week by measuring the length and width with calipers. Tumor volume was determined using the following formula: volume $=0.5 \times$ length $\times$ width $^{2}$. The tumors were collected at the time-point of 5 weeks, weighed and photographed. All experimental procedures involving animals were performed in accordance with the National Institute of Health Guide for the Care and Use of Laboratory Animals, with the approval of the Scientific Investigation Board of the Second Military Medical University (Shanghai, China).

\section{Statistical analysis}

All data are expressed as the mean \pm standard deviation from at least three independent experiments. Statistical evaluation was performed using Student's $t$-test. The patient survival analysis was performed using the log-rank (Mantel-Cox) test. The association between TRIM11 expression and p53 levels in patient samples was performed using Spearman 's correlation. $P<0.05$ was considered significant.

\section{Results}

Increased TRIM11 expression is frequently detected in HCC, and correlates with poor patient survival

To detect the vital role of TRIM11 in HCC, we first analyzed the microarray data regarding TRIM11 expression in the Oncomine database. As shown in Fig. 1A, the mRNA expression level of TRIM11 was significantly increased in human HCC samples when compared with the adjacent normal hepatic tissues. We also found that TRIM11 copy number in cancer is much higher than that in normal tissues (Fig. 1B). Moreover, high TRIM11 copy number is strongly associated with poor prognosis of HCC patients (Fig. 1C). To further confirm the results from microarray analysis in the Oncomine database, we performed real-time PCR and western blot experiments using ten human HCC specimens and the matched normal tissues. The realtime PCR experiments showed that seven tumor specimens expressed significantly higher TRIM11 levels, while two tumor specimens had comparable TRIM11 levels and one tumor specimen showed decreased TRIM11 levels compared with their matched normal tissues (Fig. 1D). Seven of ten HCC tumor samples showed obviously increased protein levels of TRIM11 when compared with their paired normal tissues (Fig. 1E). These data showed that TRIM11 is up-regulated in human HCC specimens, indicating a positive correlation of TRIM11 expression with HCC.

We then analyzed TRIM11 expression by IHC in 60 HCC tumor tissues and their matched normal tissues, and performed statistical analysis of TRIM11 staining using integral optical density (IOD). As shown in Fig. 2A and 2B, the intensity of TRIM11 staining in HCC tumor tissues was significantly increased compared with that in matched normal tissues. We also analyzed TRIM11 expression in HCC cell lines and found that the mRNA and protein levels 


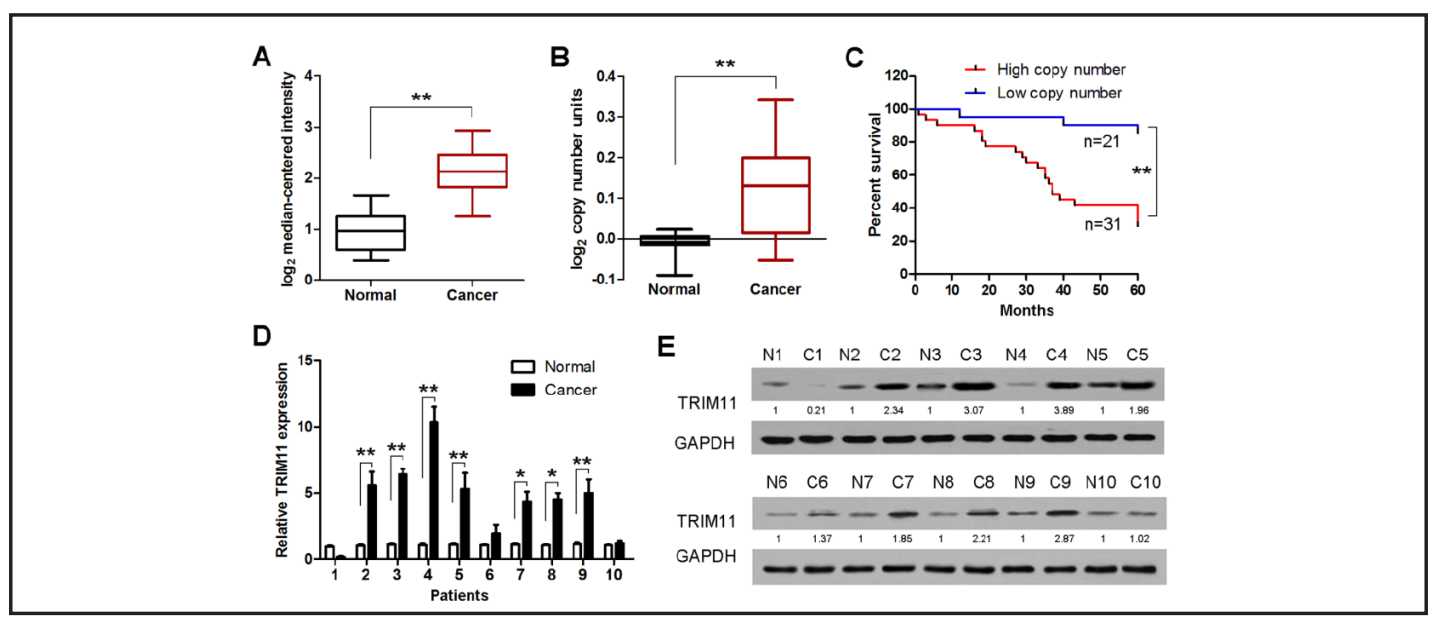

Fig. 1. The levels of TRIM11 expression in HCC tissues and cell lines. (A) TRIM11 mRNA expression levels from the Oncomine database showed that expression was much higher in HCC cancers than normal tissues. Ten samples of normal tissue and 35 cancer tissues were analyzed. Data are from the published study [27]. (B) TRIM11 copy number was much higher in HCC cancers than normal tissues, shown by analysis of 26 normal tissues and 26 cancer tissues. Data are from the published study [28]. (C) TRIM11 copy number negatively correlated with patient survival $(n=31$ in the TRIM11 high copy number, $n=21$ in the TRIM11 low copy number). Data are from the published study [28]. (D) TRIM11 mRNA levels were detected by realtime PCR in ten tumor samples and their matched normal tissues. (E) TRIM11 protein levels in ten tumor samples and their matched normal tissues. ${ }^{*} \mathrm{P}<0.05,{ }^{* *} \mathrm{P}<0.01$.

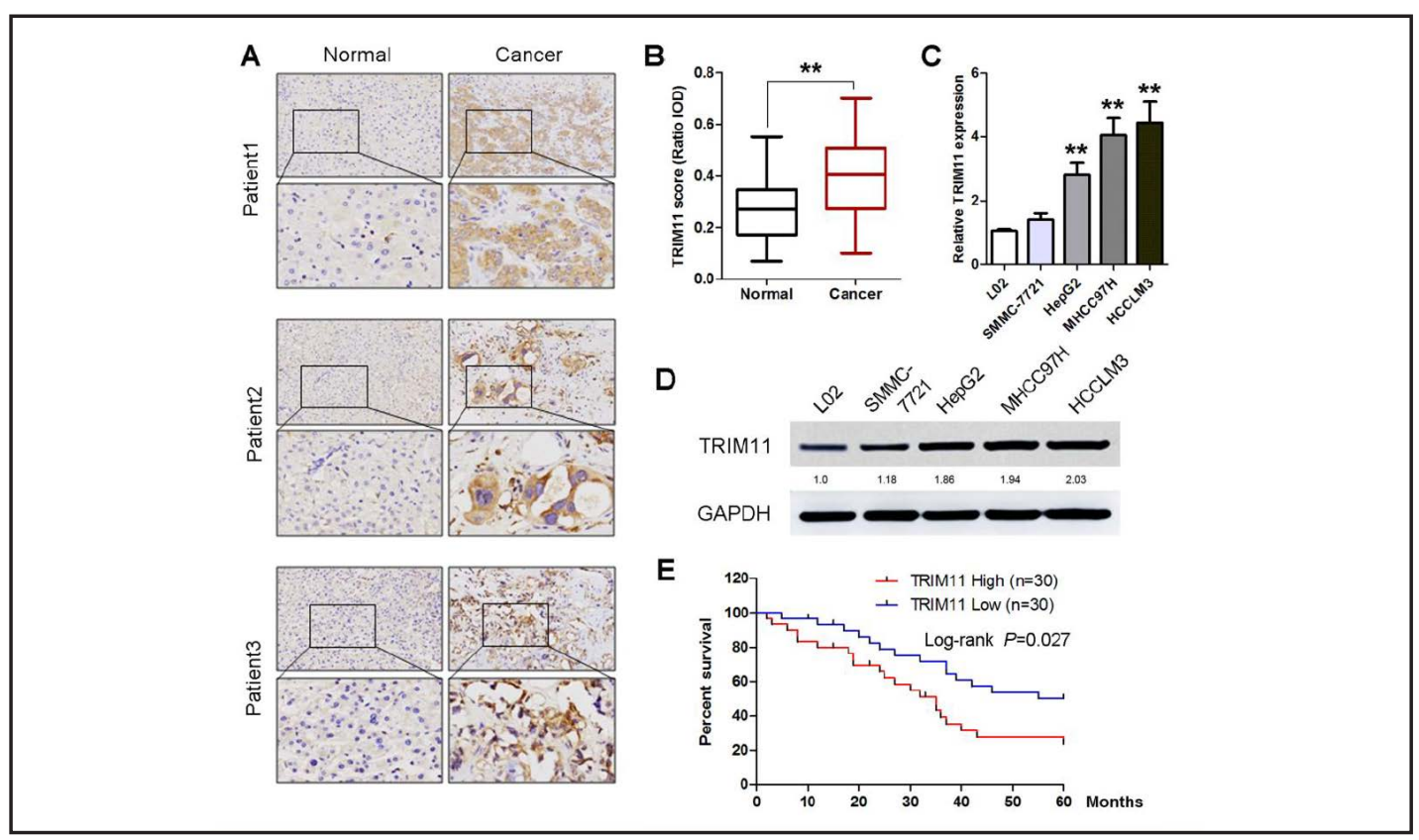

Fig. 2. TRIM11 expression is increased in HCC clinical samples and correlates with poor survival in patients. (A) Representative IHC staining of TRIM11 in HCC cancer tissues and matched normal tissues. A total of 60 HCC samples were stained and analyzed. Original magnification, 200× and 400×. (B) Quantitative analysis of TRIM11 staining. IOD, integral optical density. (C) TRIM11 mRNA levels in a normal human hepatic cell line (L02) and in HCC cell lines (SMMC-7721, HepG2, MHCC97H and HCCLM3) were analyzed by real-time PCR. (D) TRIM11 protein levels were analyzed by western blotting. (E) High TRIM11 mRNA levels were strongly associated with poor survival in HCC patients. ${ }^{*} \mathrm{P}<0.05$, ${ }^{* *} \mathrm{P}<0.01$. 
A
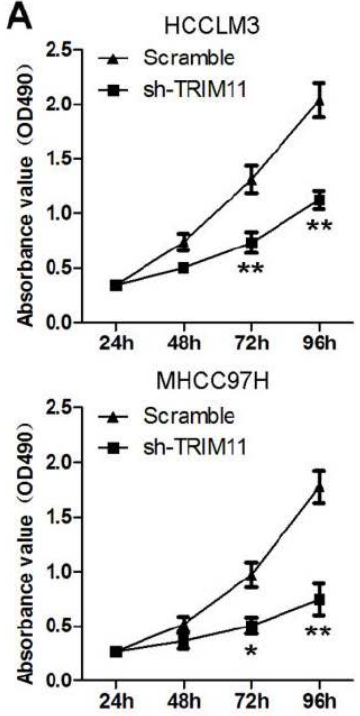

E

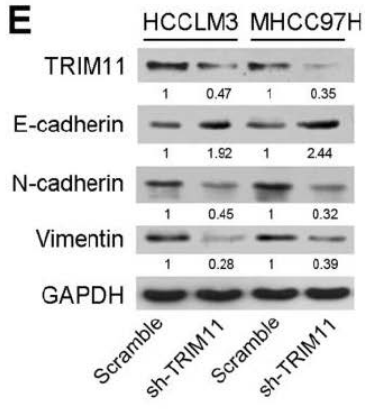

B
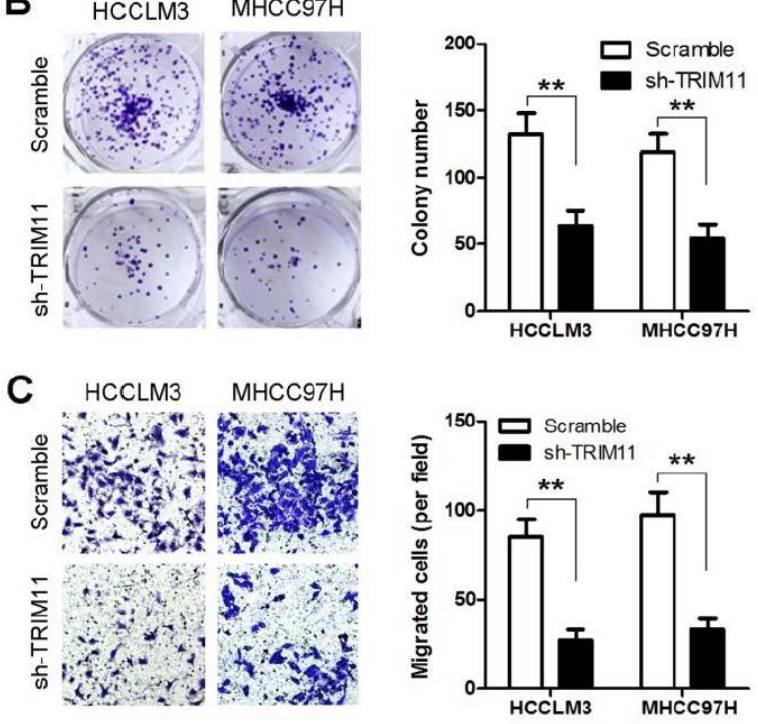

D
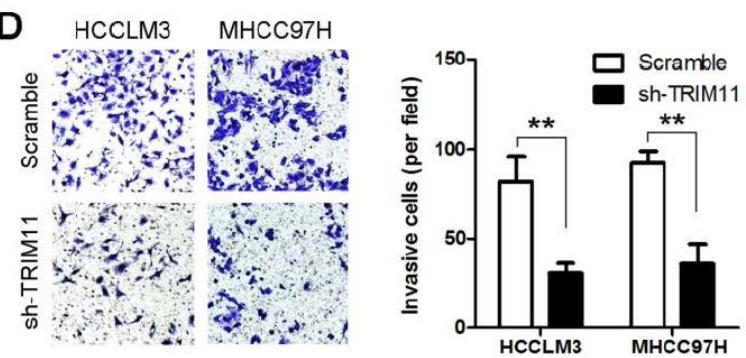

Fig. 3. Downregulation of TRIM11 suppresses HCC cell proliferation, colony formation, migration, invasion and EMT. (A) Cell viability of HCCLM3 and MHCC97H cells expressing sh-TRIM11 and scramble was examined by MTT assay. (B) Cell proliferation was analyzed by colony formation assay and analyzed in the indicated cells. (C) Cell migration was evaluated by Transwell ${ }^{\mathrm{TM}}$ migration assay. (D) Cell invasion was analyzed by Transwell invasion assay. (E) The protein levels of TRIM11, E-cadherin, N-cadherin and Vimentin in the indicated cells were analyzed by western blotting. ${ }^{*} \mathrm{P}<0.05,{ }^{* *} \mathrm{P}<0.01$.

of TRIM11 in the cell lines HepG2, MHCC97H and HCCLM3 are much higher than the normal human hepatic cell line L02 (Fig. 2C and 2D). We classified the 60 HCC patients into two groups according to TRIM11 expression levels and found that high TRIM11 expression was strongly associated with shortened HCC patient survival (Fig. 2E). Collectively, these data demonstrate that TRIM11 may play a role in promoting HCC.

\section{Effects of TRIM11 on cell proliferation, migration, invasion and EMT}

To explore the biological function of TRIM11 in HCC, we first used a lentivirus system containing TRIM11 shRNA (sh-TRIM11) to knock down TRIM11 expression in HCCLM3 and MHCC97H cells. Results of the MTT assay showed that cell viability was significantly inhibited in these cells when TRIM11 was knocked down using sh-TRIM11 (Fig. 3A). The colony formation assay showed that the colony number was significantly decreased in both of the sh-TRIM11-expressing cell lines (Fig. 3B). Furthermore, transwell migration and invasion assays showed that TRIM11 knockdown significantly suppressed the migration and invasion of HCCLM3 and MHCC97H cells (Fig. 3C and 3D). These observations suggest that downregulation of TRIM11 suppressed in vitro proliferation, migration and invasion of HCC 
A

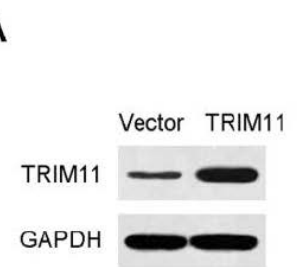

B

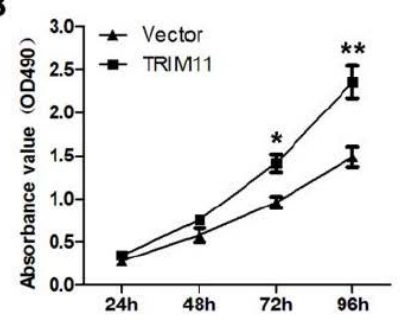

D
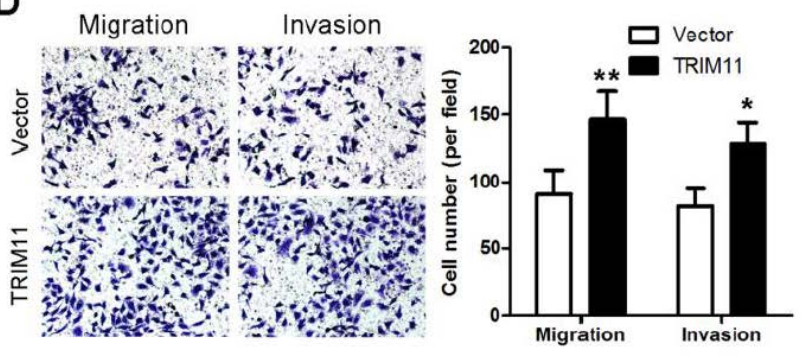
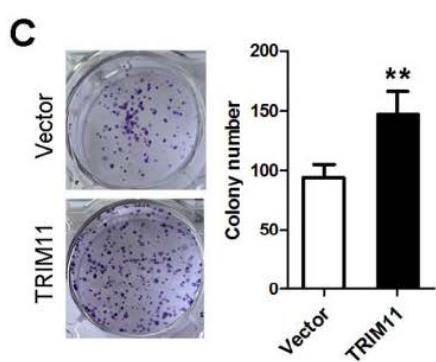

E

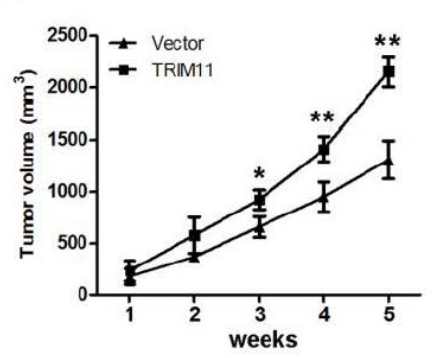

Fig. 4. The effects of TRIM11 overexpression on the proliferation, migration, invasion, and tumor growth of SMMC-7721 cells. (A) Western blot analysis of TRIM11 expression in SMMC-7721 cells infected lentivirus expressing TRIM11 or vector. Overexpression of TRIM11 significantly promoted cell proliferation (B), colony formation (C) and migration (D). (E) TRIM11 overexpression promotes tumor growth in vivo. ${ }^{*} \mathrm{P}<0.05,{ }^{* *} \mathrm{P}<0.01$.

cells. Next, we investigated the role of TRIM11 in epithelial-mesenchymal transition (EMT). As shown in Fig. 3E, the protein levels of E-cadherin were increased, while N-cadherin and Vimentin were significantly decreased in both HCCLM3-sh-TRIM11 and MHCC97H-shTRIM11 cells compared with the scramble shRNA-treated cells.

In contrast, TRIM11 overexpression in SMMC-7721 cells, which have low endogenous TRIM11 levels, significantly increased cell proliferation, colony formation, migration and invasion, compared to the negative control (Fig. 4A-4D). These results demonstrate that TRIM11 promotes several malignancy parameters in human HCC cells.

TRIM11 regulates in vivo tumor growth of HCC

To detect whether the function of TRIM11 observed in vitro corresponded to HCC tumor growth in vivo, HCCLM3 cells stably expressing sh-TRIM11 and scramble shRNA were subcutaneously injected into BALB/c nude mice. As shown in Fig. 5A, the tumors from mice bearing HCCLM3-sh-TRIM11 cells grew more slowly than those from HCCLM3-scramble shRNA cells after inoculation, and the difference in tumor volume between the two groups of mice continued to increase up to the experimental endpoint ( 5 weeks). Moreover, the difference of tumor development was also verified by the size of tumors that were excised at 5 weeks after inoculation from the two groups of mice (Fig. 5B). In contrast, the tumors in the SMMC-7721-TRIM11 group grew much faster than those in the SMMC-7721-Vecor group (Fig. 4E). We also detected the expression indexes of Ki67 in tumors using IHC to assess proliferation in vivo. As shown in Fig. 5C, the Ki67 expression level was much lower in sh-TRIM11 tumors than in scramble shRNA tumors, suggesting that TRIM11 functions to positively regulate tumor growth. In addition, we found that the expression levels of E-cadherin increased, while Vimentin expression decreased in sh-TRIM11 tumors compared with that from scramble shRNA tumors (Fig. 5D), indicating that TRIM11 promotes EMT in vivo.

\section{KARGER}


A

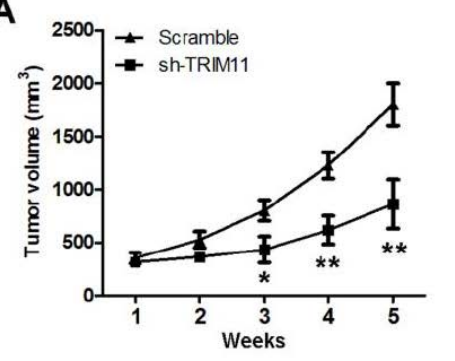

C

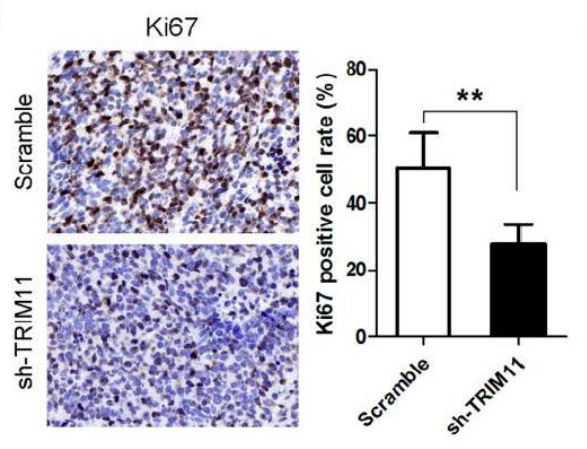

B

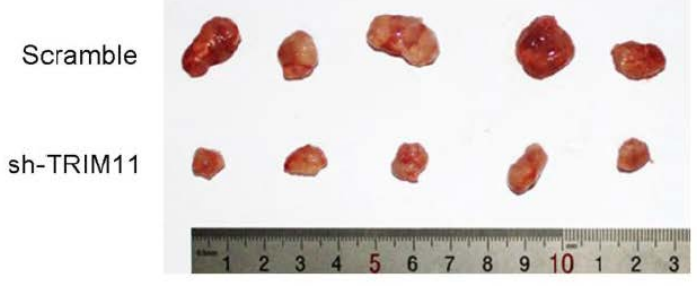

D

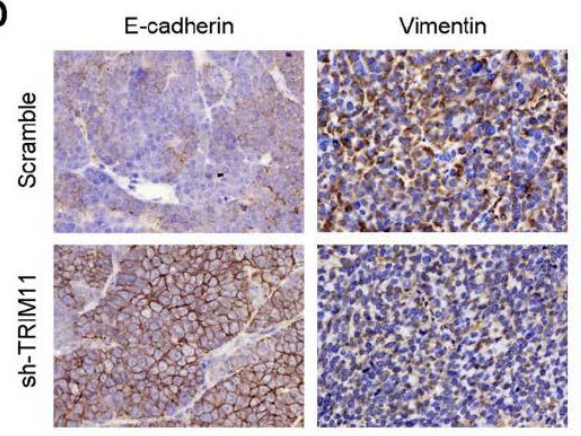

Fig. 5. TRIM11 downregulation inhibits tumor growth of HCC cells in vivo. (A) Flank tumors were established in nude mice $(n=5)$. sh-TRIM11 inhibits tumorigenicity of HCCLM3 cells in vivo. Tumor volumes were analyzed at the different time points. (B) Representative images of xenograft tumors from mice bearing HCCLM3-sh-TRIM11 cells and HCCLM3-scramble cells are shown. (C) Ki67 expression was detected by IHC in xenograft tumors. (D) Expression of E-cadherin and Vimentin was detected by IHC in xenograft tumors. ${ }^{*} \mathrm{P}<0.05,{ }^{* *} \mathrm{P}<0.01$.

TRIM11 expression correlates with $p 53$ suppression in vitro and in vivo

We next explored the molecular mechanisms under which TRIM11 promotes HCC. Given that several TRIM family members can regulate the stability or transcriptional activity of p53 [7], which is one of the most important tumor suppressors, we speculate that TRIM11 may also regulate p53 expression. To this end, we first determined the expression levels of p53 and its downstream molecules after TRIM11 knockdown. As shown in Fig. 6A and 6B, the mRNA and protein levels of p53, p21 and p27 were significantly increased, while Cyclin D1 levels were decreased in HCCLM3-sh-TRIM11 cells compared with that in HCCLM3-scramble cells, indicating that TRIM11 regulates p53 and its downstream molecule expression in vitro.

The vital role of p53 in TRIM11-induced HCC tumor growth prompted us to investigate whether the decreased tumor growth in sh-TRIM11 xenograft results from the enhancement of p53 expression. We then analyzed p53 expression levels in tumors by western blotting and IHC from two groups of mice 5 weeks after inoculation. TRIM11 knockdown was confirmed in sh-TRIM11 xenograft tumors (Fig. 6C and 6D). The expression levels of p53 were significantly increased in sh-TRIM11 xenograft tumors compared with that in scramble shRNA xenograft tumors (Fig. 6C and 6D), suggesting that p53 signaling may participate in TRIM11-induced HCC tumor growth. Furthermore, an inverse correlation was observed between TRIM11 and p53 protein in the 10 HCC samples (Fig. 6E).

TRIM11 exerts its oncogenic effects through repressing p53

To further confirm the significant role of p53 in the HCC tumor-promoting function of TRIM11, we investigated the influence of p53 knockdown on tumor growth of HCCLM3-sh- 
A

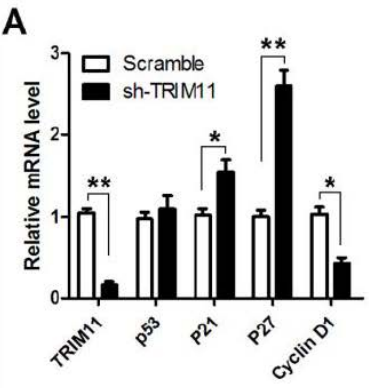

B

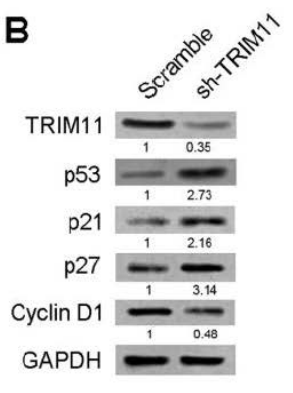

E

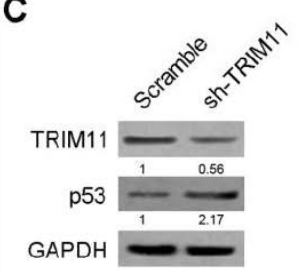

G

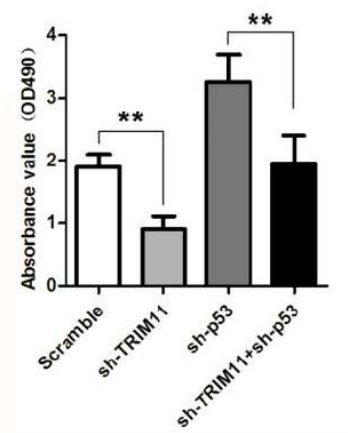

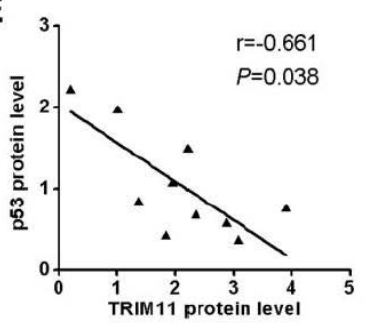

H

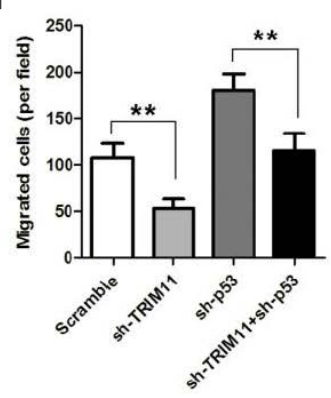

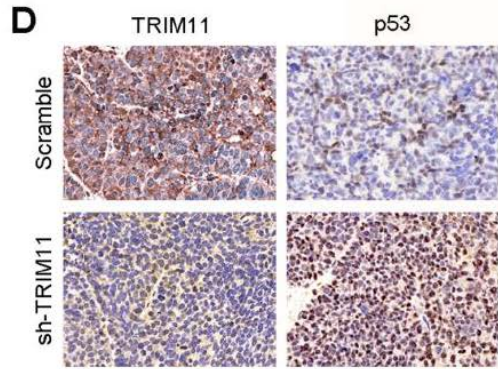

$\mathrm{F}$

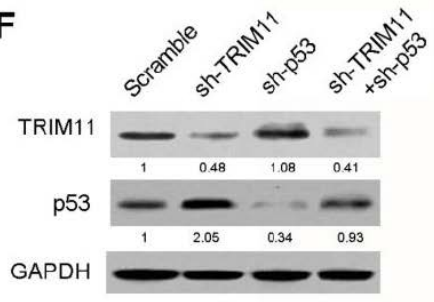

I

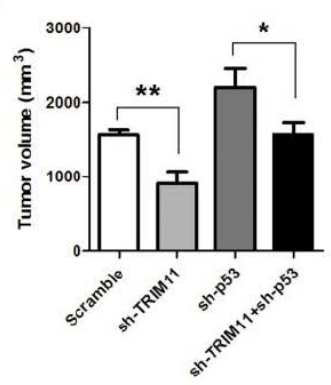

Fig. 6. TRIM11 negatively regulates p53 expression and p53 knockdown relieves the effects of sh-TRIM11 in vitro and in vivo. The mRNA (A) and protein (B) expression levels of TRIM11, p53, p21, p27 and CyclinD1 were analyzed in HCCLM3-sh-TRIM11 and HCCLM3-scramble cells. (C) The expression levels of TRIM11 and p53 in tumors inoculated by HCCLM3-sh-TRIM11 and HCCLM3-scramble cells were detected by western blotting. (D) TRIM11 and p53 expression in xenograft tumors was analyzed by IHC. (E) Spearman's correlation analysis between TRIM11 and p53 protein levels in 10 human HCC samples. (F) The expression levels of TRIM11 and p53 were analyzed by western blotting in HCCLM3 cells infected by scramble, shTRIM11, sh-p53 and sh-TRIM11+sh-p53 lentivirus. (G) Cell viability was analyzed at $96 \mathrm{~h}$ by MTT assay in the indicated cells. $(\mathrm{H})$ Cell migration was analyzed by Transwell ${ }^{\mathrm{TM}}$ migration assay. (I) Flank tumors were established in nude mice. Tumor volumes are shown $(\mathrm{n}=4)$. ${ }^{*} \mathrm{P}<0.05,{ }^{*} \mathrm{P}<0.01$.

TRIM11 cells (Fig. 6F). As shown in Fig. 6G, p53 knockdown rescued the cell proliferation ability of HCCLM3 cells, which was inhibited by TRIM11 knockdown. Moreover, p53 knockdown increased migration in sh-TRIM11-HCCLM3 cells (Fig. 6H). We next investigated whether p53 knockdown could also rescue tumor growth in vivo after inhibition in HCCLM3sh-TRIM11 xenograft mice. As shown in Fig. 6I, tumor size and volume in the sh-TRIM11 group were much smaller than that of scramble shRNA tumors. However, p53 knockdown (sh-TRIM11+sh-p53 group) promoted tumor growth, with significantly increased tumor volumes compared with that of the sh-TRIM11 group. Together, these data demonstrate that TRIM11 exerts its oncogenic role through negatively regulating p53. 


\section{Discussion}

HCC constitutes one of the most common causes of cancer-related death worldwide [19]. However, the mechanism associated with the development and progression of HCC remains unclear. In the present study, we provide experimental evidence that TRIM11 promotes the proliferation, migration, invasion and EMT of HCC in vitro and tumor growth in vivo. In addition, we found that TRIM11 promotes HCC through the p53 pathway. Thus, we suggest that TRIM11 expression in hepatic biopsy detected by RT-PCR, western blot or IHC could be a potential indicator to predict HCC behaviors when combined with various pathologic examinations, which will be useful in personalized diagnosis of HCC patients.

The biological functions of TRIM11 in HCC development and progression were explored in vitro and in vivo using HCC cell lines. Our results demonstrate that TRIM11 interference inhibits colony formation, migration and invasion of HCCLM3 and MHCC97H cells in vitro. Our studies in vivo also demonstrated the tumor-promoting role of TRIM11 in HCC, as evidenced by decreased tumor growth and Ki67 staining in tumor inoculated using shTRIM11-HCCLM3 cells. These data indicate that TRIM11 promotes HCC development and progression, which is consistent with previous studies [16,17].

The epithelial-mesenchymal transition (EMT) is a key developmental process that is activated in cancer invasion, during which epithelial cells often acquire an enhanced invasive phenotype when exposed at the front of invasive carcinoma [20-22]. In the present study, we analyzed the expression levels of EMT markers at both the mRNA and protein levels in vitro and in vivo, and found that E-cadherin (epithelial marker) increased, while $\mathrm{N}$-cadherin and Vimentin (mesenchymal markers) decreased significantly in sh-TRIM11-HCCLM3 cells and tumors, indicating that TRIM11 promotes HCC growth and progression at least partly through enhancing EMT.

p53, a known tumor suppressor, participates in various critical biological processes [23]. The p53 mutation plays crucial roles in HCC development. For example, Morris and colleagues showed that liver-specific deletion of p53 results in liver cancer (both HCC and cholangiocarcinomas) in about $41 \%$ of mice at 10 months, along with increased transforming growth factor-beta levels [24]. It is noteworthy that the expression levels of p53 are regulated by various E3 ubiquitin ligases, including TRIM proteins, such as TRIM25 and TRIM59 $[25,26]$. In the present study, we showed that TRIM11 similarly decreased the expression levels of p53 and regulated its downstream molecules in vitro and in vivo. Moreover, p53 knockdown in the sh-TRIM11-HCCLM3 cell line rescued the cell proliferation, clone formation and migration ability of HCCLM3 cells in vitro and tumor growth in vivo after inhibition by TRIM11 knockdown, indicating that TRIM11 promotes HCC tumor growth through repressing p53.

It is important to note that our data are based on human HCC cell lines inoculated into a xenograft mouse model, during which tumor development in mice was triggered and accelerated by tumor cells, while human HCC develops spontaneously in the liver. However, TRIM11 and p53 expression in HCC clinical samples and murine tissue showed a similar profile in spite of the difference between the human data and the mouse model used in our study.

In summary, our study showed that TRIM11 promotes cell proliferation, migration, invasion and EMT in vitro and in vivo. TRIM11 promotes the development and progression of HCC through regulating p53 and its downstream signals. Taken together, our data show the critical roles of TRIM11 in HCC development and progression, and provide potential therapeutic targets for HCC treatment.

\section{Acknowledgements}

This work was supported by the funds from the National Natural Science Foundation of China (81301724), Shanghai Municipal Health Bureau Youth Research Project (20134y024), 
Shanghai Charity Cancer Research Charity Jogging Project, the Peiying Program of Putuo Hospital (2016206A and 2016214B) and Department of Respiratory Medicine Development Fund of Putuo District (2016PTZK03).

\section{Disclosure Statement}

The authors declare no conflicts of interest.

\section{References}

1 Rossi L, Zoratto F, Papa A, Iodice F, Minozzi M, Frati L, Tomao S: Current approach in the treatment of hepatocellular carcinoma. World J Gastrointest Oncol 2010;2:348-359.

-2 Hato T, Goyal L, Greten TF, Duda DG, Zhu AX: Immune checkpoint blockade in hepatocellular carcinoma: Current progress and future directions. Hepatology 2014;60:1776-1782.

-3 El-Serag HB, Kanwal F: Epidemiology of hepatocellular carcinoma in the united states: Where are we? Where do we go? Hepatology 2014;60:1767-1775.

4 Ozato K, Shin DM, Chang TH, Morse HC, 3rd: Trim family proteins and their emerging roles in innate immunity. Nat Rev Immunol 2008;8:849-860.

5 Versteeg GA, Rajsbaum R, Sanchez-Aparicio MT, Maestre AM, Valdiviezo J, Shi M, Inn KS, Fernandez-Sesma A, Jung J, Garcia-Sastre A: The e3-ligase trim family of proteins regulates signaling pathways triggered by innate immune pattern-recognition receptors. Immunity 2013;38:384-398.

6 Ikeda K, Inoue S: Trim proteins as ring finger e3 ubiquitin ligases. Adv Exp Med Biol 2012;770:27-37.

7 Hatakeyama S: Trim proteins and cancer. Nat Rev Cancer 2011;11:792-804.

8 Nisole S, Stoye JP, Saib A: Trim family proteins: Retroviral restriction and antiviral defence. Nat Rev Microbiol 2005;3:799-808.

-9 Niikura T, Hashimoto Y, Tajima H, Ishizaka M, Yamagishi Y, Kawasumi M, Nawa M, Terashita K, Aiso S, Nishimoto I: A tripartite motif protein trim 11 binds and destabilizes humanin, a neuroprotective peptide against alzheimer's disease-relevant insults. Eur J Neurosci 2003;17:1150-1158.

10 Hong SJ, Chae H, Lardaro T, Hong S, Kim KS: Trim11 increases expression of dopamine beta-hydroxylase gene by interacting with phox2b. Biochem Biophys Res Commun 2008;368:650-655.

11 Tuoc TC, Stoykova A: Trim11 modulates the function of neurogenic transcription factor pax6 through ubiquitin-proteosome system. Genes Dev 2008;22:1972-1986.

12 Darshit BS, Ramanathan M: Activation of akt1/gsk-3beta/beta-catenin-trim11/survivin pathway by novel gsk-3beta inhibitor promotes neuron cell survival: Study in differentiated sh-sy5y cells in ogd model. Mol Neurobiol 2015;53:6716-6729.

13 Lee Y, Song B, Park C, Kwon KS: Trim11 negatively regulates ifnbeta production and antiviral activity by targeting tbk1. PLoS One 2013;8:e63255.

14 Yuan T, Yao W, Huang F, Sun B, Yang R: The human antiviral factor trim11 is under the regulation of hiv-1 vpr. PLoS One 2014;9:e104269.

15 Liu T, Tang Q, Liu K, Xie W, Liu X, Wang H, Wang RF, Cui J: Trim11 suppresses aim2 inflammasome by degrading aim2 via p62-dependent selective autophagy. Cell Rep 2016;16:1988-2002.

-16 Di K, Linskey ME, Bota DA: Trim11 is overexpressed in high-grade gliomas and promotes proliferation, invasion, migration and glial tumor growth. Oncogene 2013;32:5038-5047.

-17 Wang X, Shi W, Shi H, Lu S, Wang K, Sun C, He J, Jin W, Lv X, Zou H, Shu Y: Trim11 overexpression promotes proliferation, migration and invasion of lung cancer cells. J Exp Clin Cancer Res 2016;35:100.

18 Chen Y, Li L, Qian X, Ge Y, Xu G: High expression of trim11 correlates with poor prognosis in patients with hepatocellular carcinoma. Clin Res Hepatol Gastroenterol 2017;41:190-196.

19 Eggert T, Wolter K, Ji J, Ma C, Yevsa T, Klotz S, Medina-Echeverz J, Longerich T, Forgues M, Reisinger F, Heikenwalder M, Wang XW, Zender L, Greten TF: Distinct functions of senescence-associated immune responses in liver tumor surveillance and tumor progression. Cancer Cell 2016;30:533-547. 


\section{Cellular Physiology Cell Physiol Biochem 2017;44:255-266

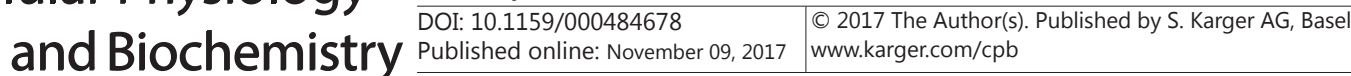

Liu et al.: Role of TRIM11 in HCC

20 Mani SA, Guo W, Liao MJ, Eaton EN, Ayyanan A, Zhou AY, Brooks M, Reinhard F, Zhang CC, Shipitsin M, Campbell LL, Polyak K, Brisken C, Yang J, Weinberg RA: The epithelial-mesenchymal transition generates cells with properties of stem cells. Cell 2008;133:704-715.

21 Zavadil J, Haley J, Kalluri R, Muthuswamy SK, Thompson E: Epithelial-mesenchymal transition. Cancer Res 2008;68:9574-9577.

22 Yang J, Weinberg RA: Epithelial-mesenchymal transition: At the crossroads of development and tumor metastasis. Dev Cell 2008;14:818-829.

-23 Yang CK, Yu TD, Han CY, Qin W, Liao XW, Yu L, Liu XG, Zhu GZ, Su H, Lu SC, Chen ZW, Liu Z, Huang KT, Liu ZT, Liang Y, Huang JL, Mo ZN, Qin X, Li L, Xiao KY, Peng MH, Winkle CA, O’Brien SJ, Peng T: Genome-wide association study of mki67 expression and its clinical implications in hbv-related hepatocellular carcinoma in southern china. Cell Physiol Biochem 2017;42:1342-1357.

24 Morris SM, Baek JY, Koszarek A, Kanngurn S, Knoblaugh SE, Grady WM: Transforming growth factor-beta signaling promotes hepatocarcinogenesis induced by p53 loss. Hepatology 2012;55:121-131.

-25 Zhang P, Elabd S, Hammer S, Solozobova V, Yan H, Bartel F, Inoue S, Henrich T, Wittbrodt J, Loosli F, Davidson G, Blattner C: Trim25 has a dual function in the p53/mdm2 circuit. Oncogene 2015;34:57295738.

26 Zhou Z, Ji Z, Wang Y, Li J, Cao H, Zhu HH, Gao WQ: Trim59 is up-regulated in gastric tumors, promoting ubiquitination and degradation of p53. Gastroenterology 2014;147:1043-1054.

27 Wurmbach E, Chen YB, Khitrov G, Zhang W, Roayaie S, Schwartz M, Fiel I, Thung S, Mazzaferro V, Bruix J, Bottinger E, Friedman S, Waxman S, Llovet JM: Genome-wide molecular profiles of hcv-induced dysplasia and hepatocellular carcinoma. Hepatology 2007;45:938-947.

-28 Guichard C, Amaddeo G, Imbeaud S, Ladeiro Y, Pelletier L, Maad IB, Calderaro J, Bioulac-Sage P, Letexier M, Degos F, Clement B, Balabaud C, Chevet E, Laurent A, Couchy G, Letouze E, Calvo F, Zucman-Rossi J: Integrated analysis of somatic mutations and focal copy-number changes identifies key genes and pathways in hepatocellular carcinoma. Nat Genet 2012;44:694-698. 